\title{
Worst-case evaluation for integrated chassis control systems
}

\author{
Youseok Kou ${ }^{\mathrm{a}}$, Huei Peng ${ }^{\mathrm{a}}$ and DoHyun Jung ${ }^{\mathrm{b} *}$ \\ ${ }^{a}$ Department of Mechanical Engineering, University of Michigan, Ann Arbor, USA; \\ ${ }^{b}$ Body \& Chassis Engineering Center, Korea Automotive Technology Institute, South Korea
}

(Received 30 July 2007; final version received 23 January 2008)

\begin{abstract}
A simulation-based worst-case evaluation (WCE) method for evaluating the performance of integrated chassis control (ICC) systems is reported in this paper. The ICC controller was developed based on balanced objectives in controlling vehicle motions, with carefully selected thresholds and targets. A trajectory optimisation problem which is defined to identify the worst-possible excitation (e.g., steering) to the vehicle with ICC is solved via nonlinear programming (NLP) approach. Generation of initial points is based on existing standard tests, engineering practice and linear control theories. Vehicle response under the ICC control is evaluated using the CarSim software. The effectiveness of the ICC system was examined by applying WCE procedure. In addition, WCE results provided useful insight for improving the ICC design, which can be incorporated into the overall ICC design and evaluation process.
\end{abstract}

Keywords: worst case evaluation, integrated chassis control, active safety control, rollover

\section{Introduction}

The performance of vehicles is frequently assessed by government agencies through welldefined standard tests. The test results are then published through New Car Assessment Programs, which has become critical in consumer purchase decisions. In the US, rollover propensity is assessed through a 5-star rating system, which is based on static stability factor plus a correction based on fishhook test. The fishhook maneuver is selected by National Highway Transportation Administration (NHTSA) based on objectivity, repeatability, performability and discriminatory capability [1]. Starting from the late 1990's, electronic stability control (ESC) systems quickly penetrate the market as an important active safety device. Car companies soon realised ESC is a relatively cost-effective way to boost the rollover star rating of a SUV or light trucks. For example, if wheel lift-off occurs during the dynamic fishhook testing, the star rating may be reduced from the vehicle's static-stability-factor (SSF) star designation to a lower rating. Instead of redesigning the vehicle chassis or weight distributions, ESC can be calibrated to avoid wheel lift-off and thus improve the rollover star rating.

NHTSA now faces a new and difficult problem in designing a simple, repeatable and reliable way to assess the performance of vehicles with smart chassis control systems. The problem

*Corresponding author. Email: ykou@umich.edu

ISSN 0042-3114 print/ISSN 1744-5159 online

(C) 2008 Taylor \& Francis

DOI: $10.1080 / 00423110801939196$

http://www.informaworld.com 
is analogous to assessing the learning of students. Traditional 'standard test' procedure is akin to announcing the exam questions ahead of time, and then trying to assess learning by grading the exam papers. Is it possible some 'students' may do a great job answering the exam questions but otherwise learn very little about the rest of the course material? With 'students' armed with advanced chassis control systems which can be easily tuned for any pre-announced standard test, the teacher (NHTSA) needs to find a revolutionary way to assess learning (safety performance). What we present in this paper, is a new 'testing' concept, which will reflect the safety performance of vehicles with active control under a broad range of maneuvers, instead of only under a couple of pre-defined maneuvers.

This new testing method, we believe, needs to have three major characteristics: (1) The test maneuver cannot be one-size-fit-all. Instead, it needs to create customised test maneuvers for each vehicle; (2) The test needs to be simulation-based, instead of experiment-based; and (3) The test needs to be based on comprehensive and rich test maneuvers; instead of relying on a handful of test maneuvers. These three characteristics are discussed separately in the following.

The current standard test practice also faces another major hurdle: experimental evaluations are, by nature, expensive, time-consuming and with low-repeatability because of the large number of uncontrolled variables and parameters such as tyre wear and road friction.

For vehicles with active safety systems, ensuring their functionality under extreme circumstances is a critical issue. Large test matrices can be defined to evaluate the active safety systems iteratively. To avoid time-consuming field testing, computer simulations can be used to systematically search for worst-cases situations, i.e., potential cases when the active safety systems fail to perform satisfactorily. The worst-case evaluation (WCE) methodology is an emerging field which has the potential to accelerate the development of vehicle active safety systems [2], by replacing lengthy field tests and calibration with elaborative numerical simulations. The problem of worst-case disturbance generation is closely related to the robust control problem, with the later focusing on the control design rather than disturbance generation. The solution of the Hamilton-Jacobi-Bellman equations is derived by variational calculus as the solution of the worst-case problem [3]. The worst bounded inputs of linear systems are defined by determining the worst inputs derived from the impulse responses [4]. WCE for ground vehicles and their control system had been attempted in the literature [5,6]. The focus of ref. [5] was rollover and jackknifing of articulated vehicles using the worst-case evaluation methodology. The approach is based on the dynamic game theory that control inputs and disturbances inputs compete to obtain optimal solutions, which leads to a two-player problem. The approach in ref. [6] is numerical in nature, with the vehicle plus its control system treated as a modified dynamic system. The one-player problem is solved numerically through the iterative dynamic programming method.

The WCE problem solved in this paper is similar to that of ref. [6]. It aims to solve the worst-case disturbance problem as a one-player problem, with the driver steering input treated as the only disturbance signal trying to optimise a selected cost function. The first step in the WCE process involves the development of a proper simulation model. Vehicle models suitable for integrated classic control (ICC) design and evaluation must be accurate enough under extreme maneuvers and in the meantime easy to be integrated with ICC controller and the WCE optimisation software. Because ICC systems operate under near-incident conditions, the accuracy of the model under severe maneuvers is crucial. In the ICC design, we focus on the integration of a differential braking function ESC and a suspension function (continuous damping control, (CDC)). This relatively simple ICC configuration makes it easier to study the integration and interaction of the vehicle control functions. The core of the WCE process is the development of a numerical method for the relatively complicated vehicle model. The optimisation method needs to be fast and yet achieves acceptable convergence under 
- Development of vehicle model

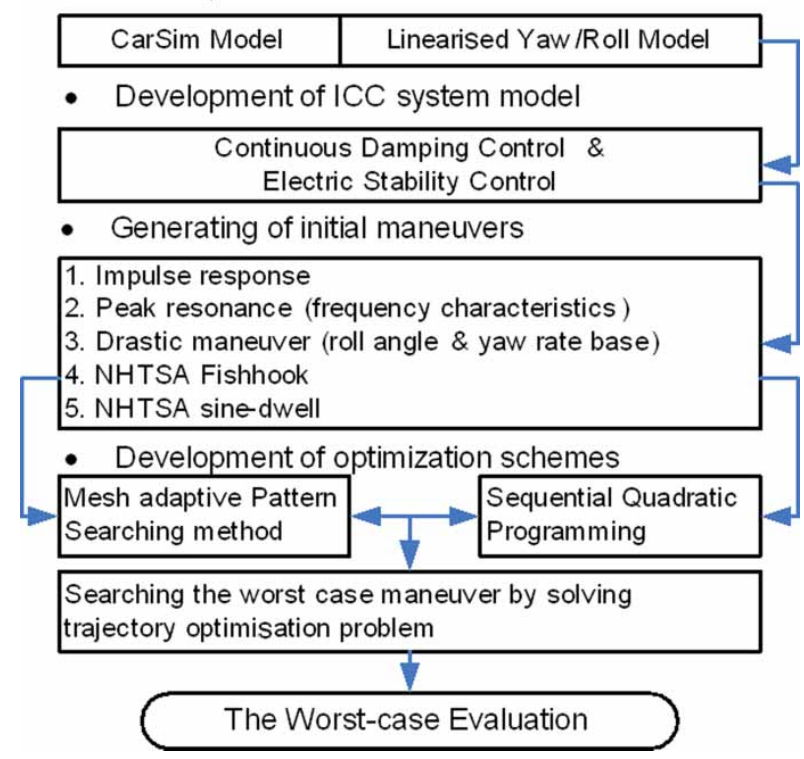

Figure 1. Diagram of the worst case evaluation process.

nonlinear dynamics and constraints. Based on previous research results, we decided to apply well-developed local search methods instead of global methods such as dynamic programming. In this paper, two numerical methods based on mesh adaptive direct searching (MADS) and sequential quadratic programming (SQP) are selected. Good initial point generation for effective local searching by the developed numerical method must be followed. These overall processes for WCE are shown in Figure 1.

\section{Design of ICC system}

The ICC is designed for improved ride comfort, lateral stability, side-slip control, yaw control, rollover prevention and wheel slip control [7]. The ICC control was designed at two levels. At the upper level, the desired suspension damping and brake torques are calculated based on yaw, side slip, roll and ride considerations. The servo control for the braking control is realised based on a sliding mode control technology, which is modified slightly to avoid the complex nonlinear form [8]. The braking forces are obtained by using the tyre ellipse concept [9]. This ICC system is intended to emulate typical functions of a production ICC, and is designed to be modular so that additional control systems can be added.

\subsection{CDC system}

The CDC system controls the sprung mass motion by changing the setting of the variable dampers using solenoid valves. The control algorithm uses information such as vertical acceleration and velocity, and steering input to manifest the behaviour of the vehicle and the intention of the driver. In this paper, the focus is assumed to be rollover prevention, which aims to stabilise vehicle motion resulted from driver's steering during high-speed cornering [10]. The 
activation of lateral stability control is based on vehicle lateral acceleration, estimated from the bicycle model:

$$
\hat{a}_{y}=\delta \cdot u_{x}^{2} \cdot\left(1+\left(u_{x} / u_{c h}\right)^{2}\right)^{-1} \frac{1}{L}
$$

This estimated acceleration is a better signal to use than that from an accelerometer because of its predictive nature and because it is less vulnerable to road grade and cross-talk disturbances. The quality of the estimation provided by Equation (1) depends on the accuracy of our estimate of characteristic speed, which depends on tyre cornering stiffness. In addition, the effect of road bank angle is not considered. The estimated acceleration, $\hat{a}_{y}$ is compared with a threshold value, $a_{y}^{\text {thr }}$ to check the severity of rollover threat. If $\hat{a}_{y}$ is larger than $a_{y}^{\text {thr }}$, the lateral stability control is activated. Lateral stability control gain, $K_{\text {lat }}$ proportional to vehicle speed is calibrated and then the desired damping torque calculated as $T_{\text {lat }}^{\mathrm{CDC}}=K_{\text {lat }} \cdot \hat{a}_{y}$ are applied to CDC dampers at the four corners. The overall procedure is described in the flow chart in Figure 2.

\subsection{ESC system}

The ESC system controls the braking forces of the four tyres to stabilise vehicle lateral, yaw and roll motions. In the following we will define the major functions of the ESC system and the target state values. The general ESC system (which includes the ABS functions) includes four control objectives. In the order of descending priority, these four functions are: wheel slip control, rollover prevention control, yaw control and side slip control. The wheel slip control is imposed to limit magnitude of the wheel slip to maintain $0.1 \sim 0.2$. The other three control functions are active when threshold values are exceeded (roll and side slip) or when the error is large (yaw). A desired yaw rate is calculated first from linear vehicle steady-state cornering [11]:

$$
r_{d}=\frac{\delta \cdot u_{x}}{1+\left(u_{x} / u_{c h}\right)^{2}} \cdot \frac{1}{L}
$$

This value is then saturated based on a nominal road friction value and vehicle forward speed

$$
\left|r_{d}\right| \leq r_{\lim }=\left|\frac{\mu \cdot g}{u_{x}}\right|=\left|\frac{a_{y}}{u_{x}}\right|
$$

The obtained desired yaw rate is used to calculate a yaw error, $\Delta r=r_{d}-r_{m}$ where $r_{m}$ is measured yaw rate, based on which a yaw control command will be calculated. The limit for the side slip angle, $\beta_{\text {thresh }}$ is chosen to be five degrees. When this threshold value is exceeded, yaw moment will be requested to reduce the magnitude of the side slip angle to maintain driver's control authority.

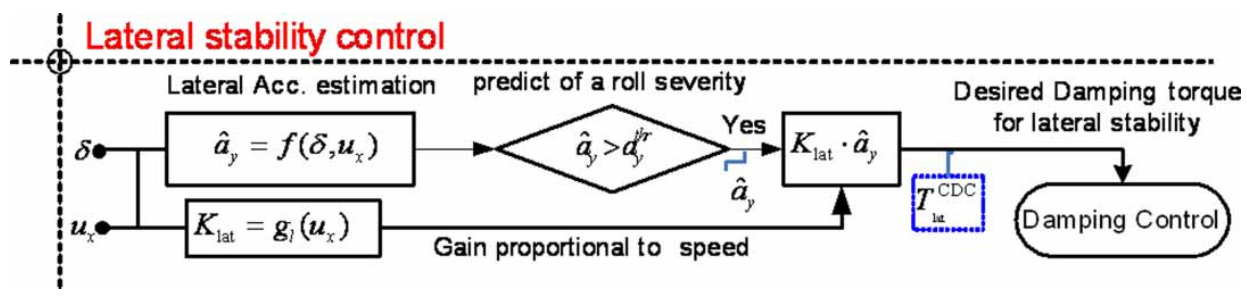

Figure 2. Flow chart of the CDC algorithm. 
To improve the responsiveness of the control system, predicted vehicle roll rate, instead of measured vehicle roll rate, is used. The predicted roll rate is calculated from

$$
\begin{aligned}
\dot{\phi}_{p}(t) & =\dot{\phi}(t+\tau)=\dot{\phi}(t)+\ddot{\phi} \cdot \tau \\
& =\dot{\phi}(t)+\left(-\frac{k_{\phi} \cdot \phi(t)+b_{\phi} \cdot \dot{\phi}(t)}{I_{x x}}+\frac{m h_{R c}}{I_{x x}} \cdot a_{y}\right) \cdot \tau
\end{aligned}
$$

where $t$ is the present time and $\tau$ is the prediction time. The roll rate is predicted based on roll rate at the present time and the roll acceleration. The roll acceleration information is estimated from a simple roll dynamic model. The overall ESC control logic is shown in Figure 3. The desirable yaw rate is first inferred from steering input and forward speed, which is saturated according to Equation (3). In parallel, the side slip threshold and the critical roll rate of the vehicle are derived according to the dynamic characteristics of the vehicle. The differences between yaw rate, side slip and roll rate and their threshold values are then calculated. If the difference is larger than the threshold gaps, $\Delta \beta_{\mathrm{thr}}, \Delta r_{\mathrm{thr}}$ and $\Delta \dot{\phi}_{\mathrm{thr}}$, the corresponding control module is activated. The desirable brake force obtained from the servo controller is passed onto the brake system. The controller detects the vehicle turning direction based on the direction of the lateral acceleration to select the wheels to be braked. The brake force is finally regulated by wheel slip control to prevent wheel lock-up, which is based on ABS system.

Before the CDC and ESC control commands are sent to the servo loop, an ICC master needs to determine the final control command based on prioritised control objectives. The ESC control command will take priority over that of CDC and the individual control system is operated in its priority sequence as explained above. Integration strategy in this paper is based on the safety priority of the control elements.

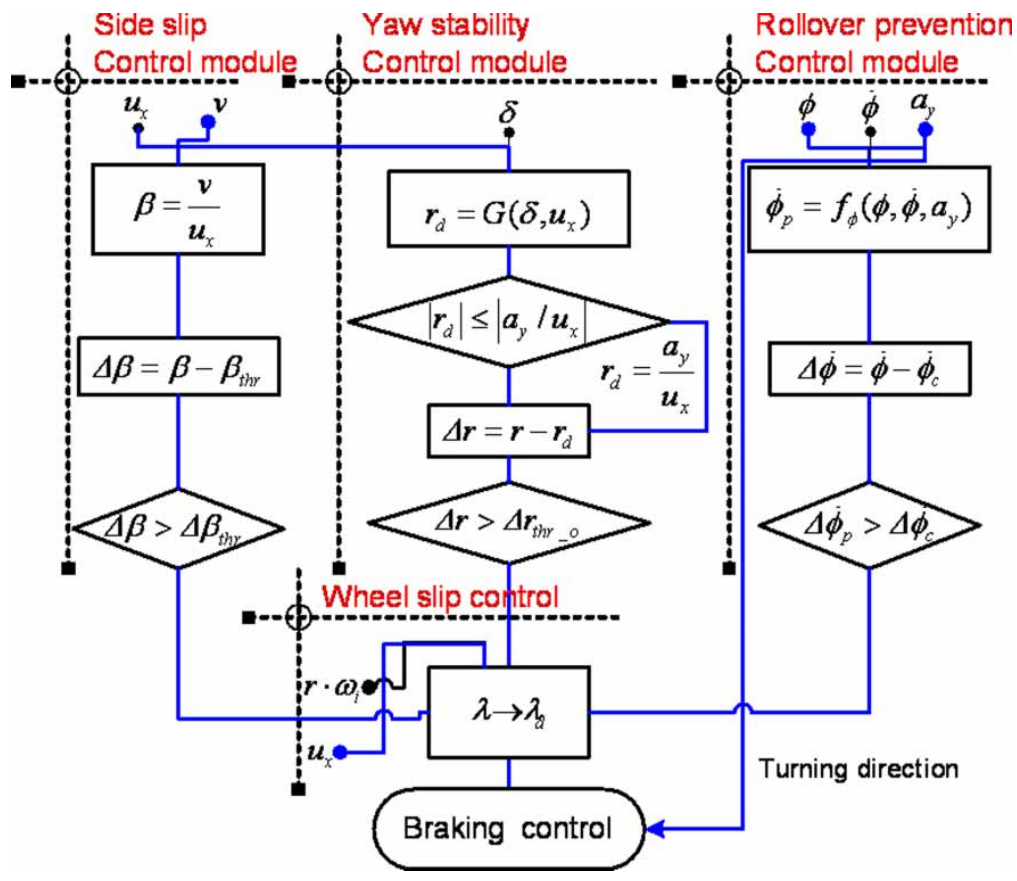

Figure 3. Flow chart of the ESC control algorithm. 


\section{The worst-case evaluation (WCE)}

The WCE process is a good alternative to the current experiments-based evaluation process for future vehicles, especially when they are equipped with active safety devices. The WCE method is a simulation-based evaluation process that identifies weaknesses of a vehicle through extensive numerical search. The simulation-based approach eliminates the effect of human uncertainties. In addition, it allows a wide variety of scenarios, including those that are not feasible or too costly in field testing. Through extensive numerical search, WCE challenges the vehicle with a large set of severe maneuvers and is a valuable asset in the development of active safety systems.

Mathematically, WCE can be formulated as a trajectory optimisation problem, which searches for the driver's maneuvers that maximise a cost function, e.g., the 2-norm of vehicle roll angle throughout the optimisation horizon. The numerical machine of WCE must be able to accommodate problems with one or more of the following features: (i) nonlinear problems with complex numerical subroutines (e.g., CarSim, Adams, etc.); (ii) problems with equality and/or inequality constraints; and (iii) performance index in non-accumulated form (e.g., infinity norm).

The WCE problem is set up as follows. The time horizon is discretised into grid points

$$
t_{0}=\tau_{1}<\cdots<\tau_{N-1}<\tau_{N}=t_{f}
$$

where $t_{0}$ : intial time $t_{f}$ : final time. The disturbance (e.g., steering wheel angle) at these discrete time grid points, $\mathbf{w}_{\mathrm{sw}}$, are design variables to be solved for the optimisation problem but the applied input is smoothed through interpolation (Figure 4)

$$
\mathbf{w}_{\mathrm{sw}}=\left[w_{\mathrm{sw}}^{1}, w_{\mathrm{sw}}^{2}, \ldots, w_{\mathrm{sw}}^{N-1}, w_{\mathrm{sw}}^{N}\right]
$$

Based on our past experience, the Dynamic Programming method, which ensures global optimality, is not practical for high-dimension dynamic systems due to the curse of dimensionality. Based on the considerations regarding convergence and computational speed, we choose the SQP method and MADS. SQP is a local search method but is very efficient even for high-dimensional problem due to its rich development history [12] and The MADS algorithm is a generalisation of the class of Generalised Pattern Search (GPS) algorithm, a derivativefree method [13]. However, both methods are local search methods and thus global optimality

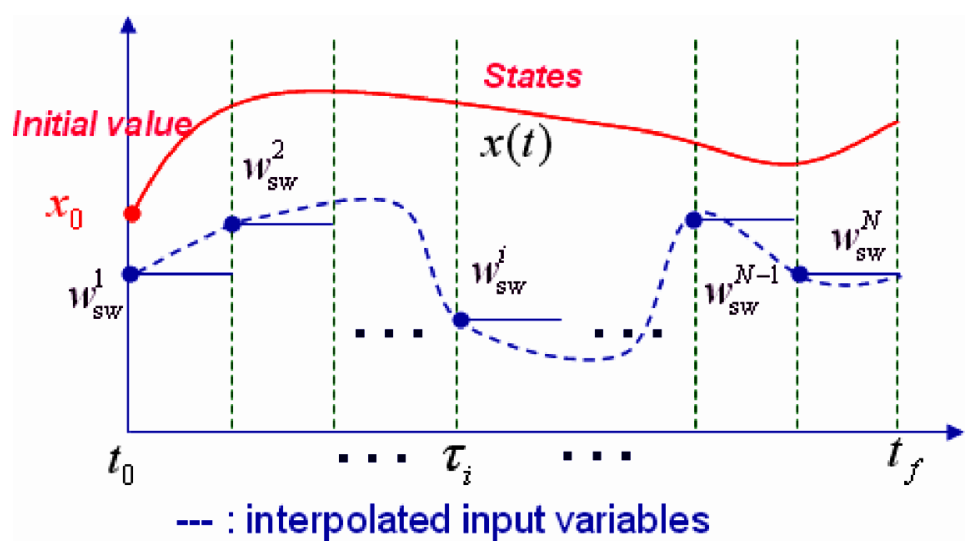

Figure 4. WCE problem setup. 
cannot be guaranteed. Therefore, it is critical to provide a rich set of initial guess of disturbance inputs.

The major pre-process of WCE program is the application of constraints and an initial point allocation. In these processing, constraints such as magnitude saturation and rate limits are imposed. The initial point generation is the most critical part of WCE. Since both numerical methods search locally, initial points that are rich and 'bad enough' are critical for reaching an array of local optimal that truly reflect the safety performance. A common practice in generating initial points for local search methods such as SQP is to start form pseudo-random points. The idea is to cover the high dimensionality of the disturbance inputs in a systematical way for richness. Some of the initial points used in our WCE program will be generated in this pseudo-random fashion. However, we put more emphasis on another generation method - to leverage existing standard test, engineering practice and controls theory. Adoption of common testing maneuvers developed by vehicle safety research group such as NHTSA and University of Michigan Transportation Research Institute (UMTRI) are very appropriate. In addition, linear systems analysis, e.g., worst allowable persistent bounded disturbance (WAPBD) [4] also provides useful insight into disturbance input generation. This concept generates worst-case input based on impulse response of a linear time invariant (LTI) system. The procedure is depicted in Figure 5. First, $g(t)$, the impulse response due to steering input is obtained. The response is trimmed at $3 \%$ steady-state error and the time span, $T$ is determined. The worst persistent disturbance, $w_{0}(t, T)$ for $t \in[0, \mathrm{~T}]$ is then obtained from $w_{0}(t, T)=$ $\operatorname{sign}\{g(T-t)\}$. Assuming that the maximum steering value is $\delta_{\max }$, then a good initial point is $\delta_{\max } \cdot \operatorname{sign}\{g(T-t)\}$.

The worst case steering inputs based on various initial points are shown in Table 1. The steering angle is constrained at the level specified in the NHTSA Fishhook test. All the searched maneuvers generate large roll angle which almost leads to rollovers. The WAPBD methodbased searching actually identifies a good initial guess which leads to rollover after the local search.

Despite the fact both SQP and MADS are local-search methods, if a large number of iterations is allowed, both methods were found to find local optimum that is quite different from the initial guess. One such example is illustrated below. In this example, the cost function to be minimised is selected to be $J=2000 /\left|\phi_{\max }\right|^{2}$. From the initial condition using WAPBD explained in the above, both MADS and SQP methods are invoked and the better result is presented as the final WCE local optimum maneuver.
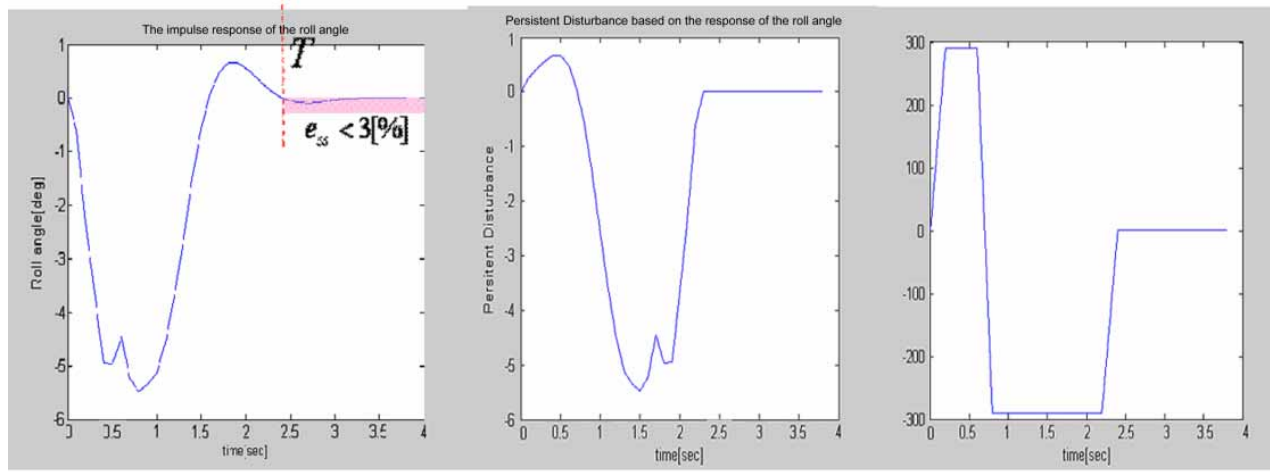

$$
g(t) \quad \Rightarrow \quad w_{0}(t, T)=g(T-t) \Rightarrow \quad \delta_{\max } \cdot \operatorname{sign}\{g(T-t)\}
$$

Figure 5. The initial point obtained from the impulse-response-based WAPBD approach. 
Table 1. Damage induced by the left-hand wheel on the first 10 sleeper and fasteners components, based on measurements and photo documentation at Bomansberget incident.

\begin{tabular}{ccc}
\hline $\begin{array}{c}\delta_{\max }: 290 \mathrm{deg} \\
\text { Middle size SUV }\end{array}$ & $\begin{array}{c}\text { Vehicle speed:80 kph } \\
\phi_{\max }: 1000 \mathrm{deg} / \mathrm{s}\end{array}$ \\
\hline & NHTSA Fishook & 14.6 \\
Initial & NHTSA sine-dwell & 13.9 \\
points & Frequecy:0.1 0.5 Hz & 13.8 \\
& WAPBD method & Rollover \\
\hline
\end{tabular}

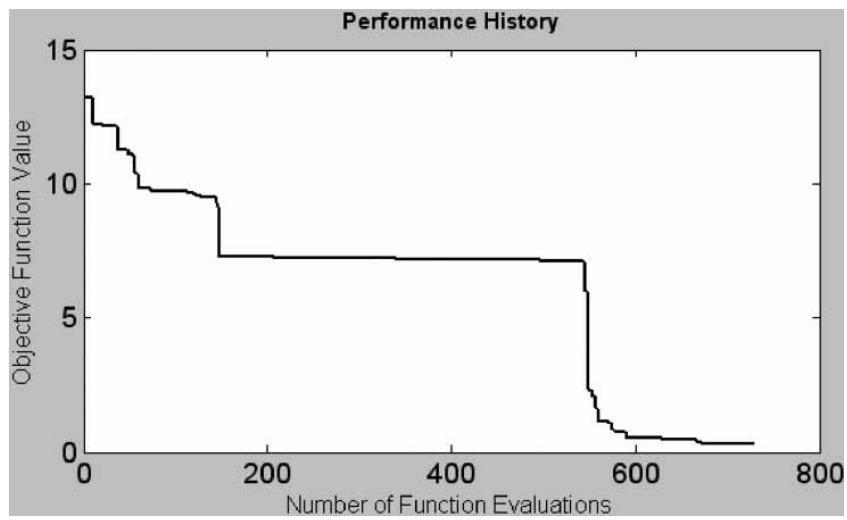

Figure 6. MADS searching history $J=2000 /\left|\phi_{\max }\right|^{2}$.

The searching history of the MADS method is shown in Figure 6. It can be seen that the solution may be stuck at a certain cost function value for extended number of iterations before it suddenly break loose and finds a better optimum point. This is typical of local search methods, which demonstrates the need to allow large numbers of iterations.

Both SQP and MADS methods were able to cause rollover, under the same initial condition as shown in Figure 7. Detailed evaluation results are shown in Table 2. The performance of the two solutions is the same $(J=0.31)$ despite the fact the steering angle and vehicle roll
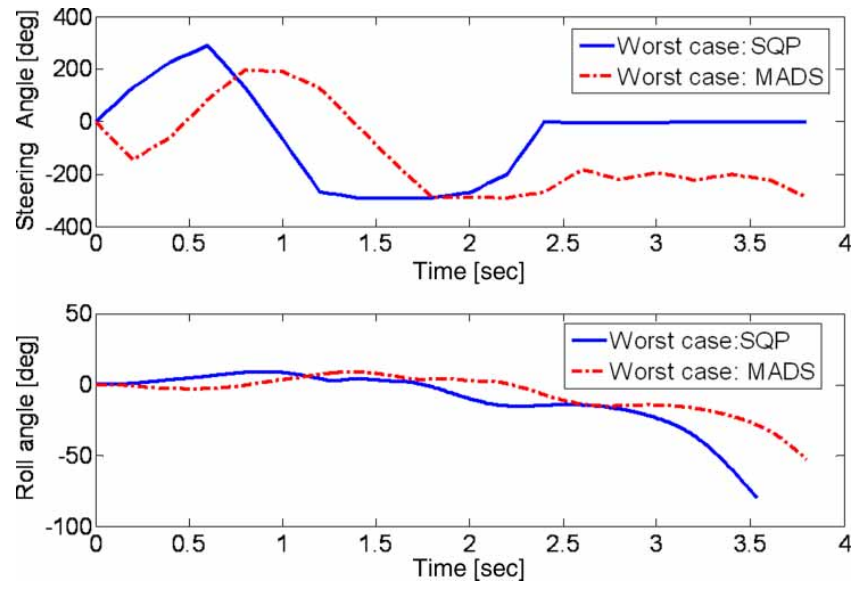

Figure 7. Worst case results from SQP \& MADS $u_{x}=82 \mathrm{kph}, \mu=0.9$. 
Table 2. WCE search results.

\begin{tabular}{lcccc}
\hline & Iteration \# & $\begin{array}{c}\text { Evaluation } \\
\text { function }\end{array}$ & $\begin{array}{c}\text { Convergence } \\
\text { tolerance }\end{array}$ & $\begin{array}{c}\text { Final cost } \\
\text { function }\end{array}$ \\
\hline SQP & 74 & 2019 & 0.001 & 0.31 \\
MADS & 349 & 729 & 0.001 & 0.31 \\
\hline
\end{tabular}
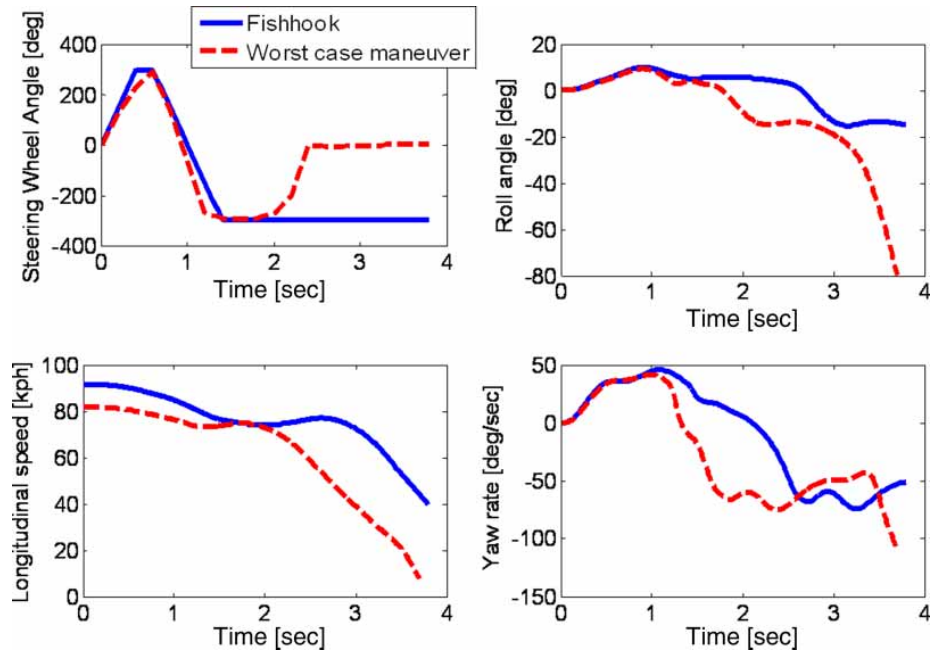

Figure 8. Comparison between standard Fishhook and the worst-case maneuver for a middle size SUV.

motions are different. This indicates that they converge to different local minima, both of which are of interest in understanding the performance of the vehicle ICC system.

The effectiveness of the obtained worst-case maneuver (from SQP) is compared against a standard rollover test, the NHTSA Fishhook test. As shown in Figure 8, the vehicle rolls over under the WCE steering but not under the standard fishhook test. What is even more interesting is that we are able to repeat the same process and achieve rollover even when the initial vehicle speed is $10 \mathrm{kph}$ lower than that of the fishhook test (Figure 8).

\section{Rollover Prevention (ROP) design based on WCE}

In this section, the ROP control strategy, one of ICC objectives is redesigned using the WCE results. The original ICC design is first evaluated using the WCE method presented in the previous section. The identified maneuver exposes potential risk of the ICC design used to obtain improved ICC.

ESC control strategies can be tuned according to the dynamic characteristics of the corresponding vehicle. Here our strategies via worst case-based reasoning are categorised into three control cases: single wheel (SW) braking, multiple wheels (MW) braking with antilock braking (ABS) and MW braking without ABS which means allowing wheel-locking. MW braking applications are used when the main goal is to regulate vehicle roll motion. SW braking at the front outside tyre is used for yaw moment stabilisation. Wheel braking at the rear outside wheel encompasses both functions with respect to the stabilisation of the vehicle planar yaw motion. One is the increasing of yaw moment via generated braking force and the other is the decreasing of yaw moment via reduced lateral force. An important decision 
point for ROP is whether wheel locking is permitted or not during the generation of braking forces. Wheel locking may be detrimental to vehicle handling response because of tyre force saturation. However, given the fact rollover events are extremely fatal, the ROP functionality may be given higher priority to other vehicle control objectives.

The NHTSA sine-dwell test specification [14] is applied to the Big SUV with high C.G, which is selected to observe the contribution of ESC ROP functionality to the target vehicle more clearly. Simulation is executed iteratively with increasing initial longitudinal speed from $80 \mathrm{kph}$. To determine ROP control strategy suitable to this big SUV, comparison tests simulation of the respective control strategy were attempted by WCE procedure regarding rollover.

First, ROP strategy based on SW braking with ABS is evaluated as Figure 9, which shows that WCE maneuver causes rollover while rollover does not occur in the sine-dwell test simulation. Through WCE procedure, we could find that SW braking fails to prevent rollover of the SUV.

The WCE procedures are conducted to investigate wheel locking effect for the ROP control. As shown in Figure 10, ROP control with ABS fails to prevent rollover because wheel locking command blocks the braking force generation at the outside wheels. MW braking without ABS succeeded in preventing rollover by generating the desired braking force, which reduce cornering forces and create compensated yaw moment regardless wheel-locking phenomena. The braking forces were generated during the wheel locking situation as shown braking control outputs $F_{x}$ and wheel locking flag in the third row of Figure 10.

Table 3 summarised important results obtained from the WCE evaluation process. As shown in this table, $\mathrm{CDC}$ is not very effective in preventing rollover but ESC is able to stabilise vehicle roll motion under NHTSA standard test maneuver. It can be seen that MW braking without ABS stabilises the vehicle better than other control strategies.

The cost function to be minimised is the same as the one used in Section $3(J=$ $2000 /\left|\phi_{\max }\right|^{2}$ ), which seeks for disturbance signal that generates large roll motions. The WCE results are obtained by 10 initial points including standard test maneuvers, the resonance characteristics analysis, WAPBD and etc. The SQP and MADS algorithms are then used to find local optimum. The results with the maximum roll angle are then reported in the table -

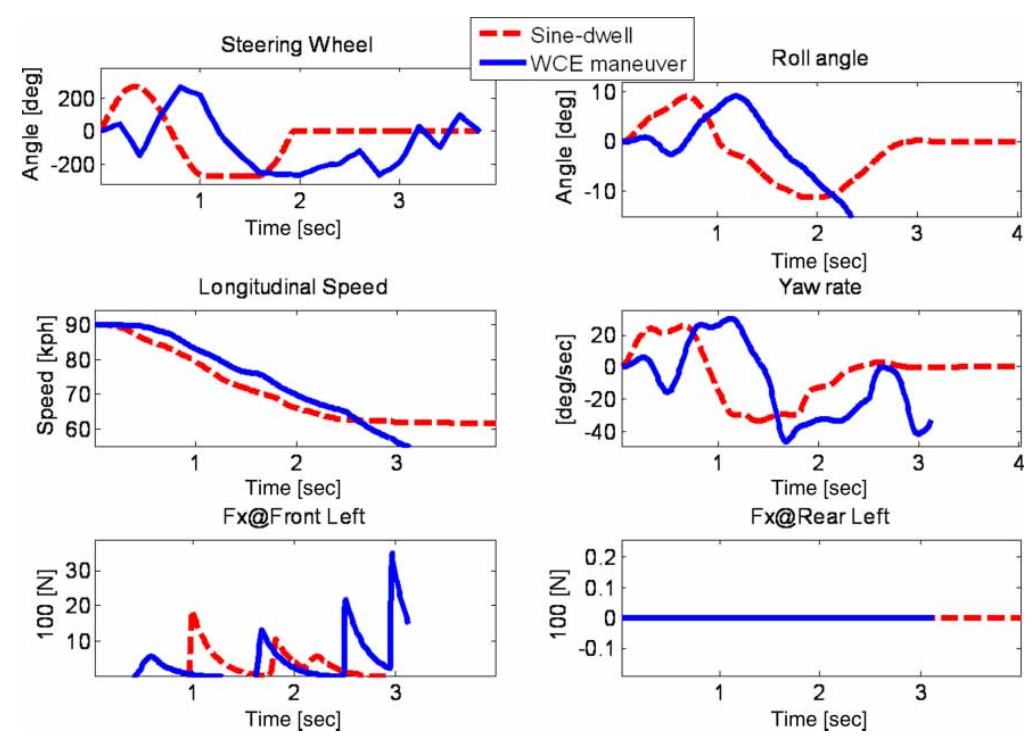

Figure 9. Comparison test simulation of NHTSA sine-dwell and WCE regarding SW braking ROP. 

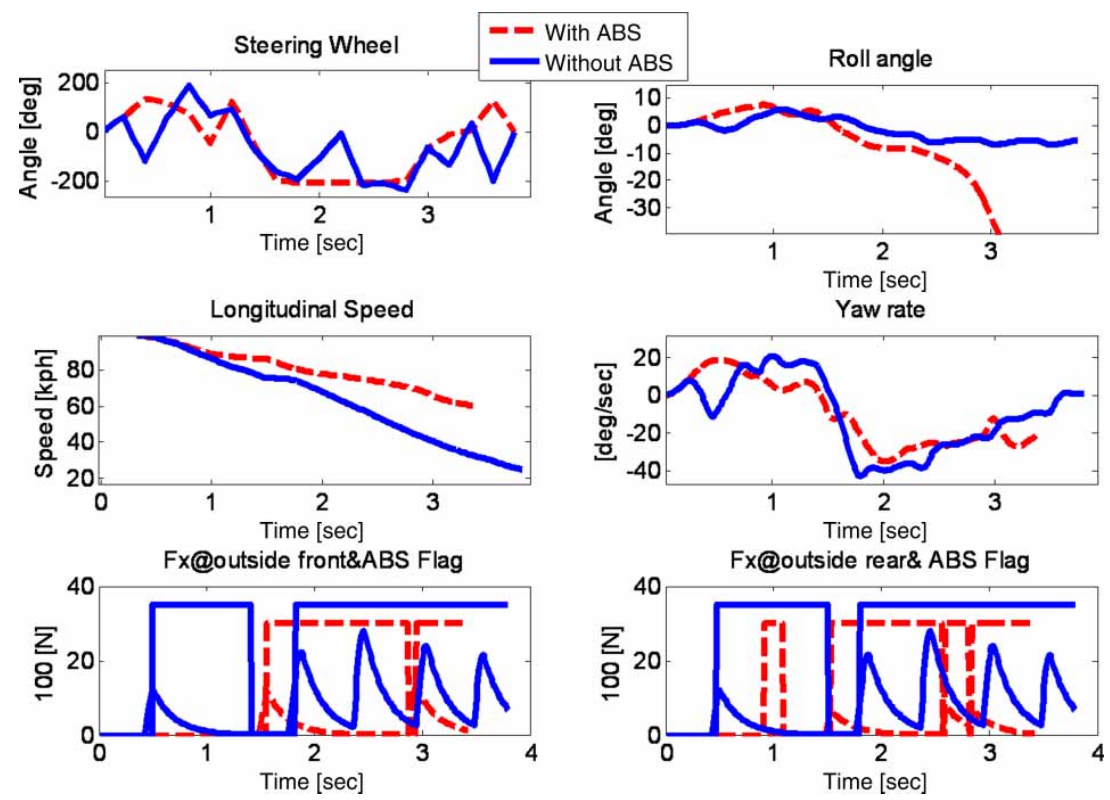

Fx@outside rear\& ABS Flag

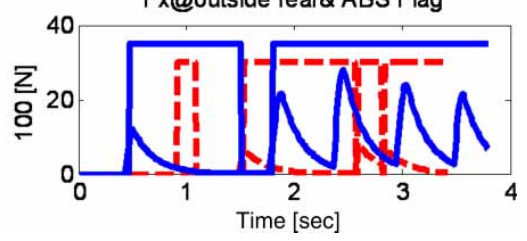

Figure 10. WCE results for comparing the performance of ROP with ABS and without ABS.

Table 3. WCE results at various initial speed and different ICC braking strategy.

\begin{tabular}{|c|c|c|c|c|c|c|c|c|c|}
\hline \multirow{2}{*}{\multicolumn{2}{|c|}{$\begin{array}{c}\left|\phi_{\max }\right| \\
\text { Control strategy/Speed }[\mathrm{kph}]\end{array}$}} & \multicolumn{4}{|c|}{ WCE } & \multicolumn{4}{|c|}{$\begin{array}{c}\text { Standard test simulation } \\
\text { (NHTSA sine-dwell and Fishhook) }\end{array}$} \\
\hline & & 80 & 90 & 100 & 110 & 80 & 90 & 100 & 110 \\
\hline \multicolumn{2}{|c|}{ CDC control* } & $\begin{array}{l}\text { Roll } \\
\text { over }\end{array}$ & $\begin{array}{l}\text { Roll } \\
\text { over }\end{array}$ & $\begin{array}{l}\text { Roll } \\
\text { over }\end{array}$ & $\begin{array}{l}\text { Roll } \\
\text { over }\end{array}$ & $\begin{array}{l}\text { Roll } \\
\text { over }\end{array}$ & $\begin{array}{l}\text { Roll } \\
\text { over }\end{array}$ & $\begin{array}{l}\text { Roll } \\
\text { over }\end{array}$ & $\begin{array}{l}\text { Roll } \\
\text { over }\end{array}$ \\
\hline \multicolumn{2}{|c|}{ One Wheel } & $\begin{array}{l}\text { Roll } \\
\text { over }\end{array}$ & $\begin{array}{l}\text { Roll } \\
\text { over }\end{array}$ & $\begin{array}{l}\text { Roll } \\
\text { over }\end{array}$ & $\begin{array}{l}\text { Roll } \\
\text { over }\end{array}$ & 10.1 & 11.2 & 13.4 & 13.13 \\
\hline \multirow[t]{2}{*}{$\begin{array}{l}\text { Multi } \\
\text { wheel }\end{array}$} & $\begin{array}{c}\text { Without } \\
\text { wheel } \\
\text { locking }\end{array}$ & $\begin{array}{l}\text { Roll } \\
\text { over }\end{array}$ & $\begin{array}{l}\text { Roll } \\
\text { over }\end{array}$ & $\begin{array}{l}\text { Roll } \\
\text { over }\end{array}$ & $\begin{array}{l}\text { Roll } \\
\text { over }\end{array}$ & 9.5 & 10.0 & $\begin{array}{l}\text { Roll } \\
\text { over }\end{array}$ & $\begin{array}{l}\text { Roll } \\
\text { over }\end{array}$ \\
\hline & $\begin{array}{l}\text { With wheel } \\
\text { locking }\end{array}$ & 10.2 & 10.8 & 10.8 & 11.3 & 9.1 & 9.9 & 10.7 & 11.0 \\
\hline
\end{tabular}

which represent our best effort to obtain the worst-case maneuver that induces rollover. For the 'standard test' columns, simulation results from NHTSA sine-dwell and Fishhook tests are obtained and the maximum roll angle is reported. When SW braking and MW braking with ABS is applied, WCE identified steering inputs that result in rollover for the target vehicle under all vehicle speeds. The performance of MW braking without ABS is able to achieve successful rollover prevention.

\section{Conclusions}

The development and evaluation of an ICC systems based on a WCE process are reported in this paper. The ICC system studied in this paper includes an ESC sub-system and a CDC sub-system. The ICC system is designed considering lateral stability control, yaw control, side-slip control, rollover prevention control and wheel slip control. The simulation-based 
WCE evaluation procedure aims to find worst possible disturbance (e.g., driver's steering input) for selected vehicle motion (e.g., rollover). Because of the high system dimension, two local search methods, SQP and MADS are used. A set of initial points compiled from common practice used in the automotive field testing, plus procedures motivated by control theories are suggested. The WAPBD theory provided an effective starting point in searching for the worst case maneuver for rollover.

The WCE process is described and its results assessing rollover prevention (ROP) performance are presented. Through this WCE, ESC's ROP performance is verified compared with CDC's. Development of ESC ROP strategy was achieved with WCE procedure. The result showed that MW braking without ABS is more robust in preventing rollover than SW braking or MW braking with ABS.

\section{References}

[1] NHTSA, NHTSA Phase IV final report, October 2002.

[2] W. Ma and H. Peng, A worst-case evaluation method for dynamic systems, ASME J. Dyn. Syst. Measure. Control 121(2) (1999), pp. 191-199.

[3] T.T. Georgiou and I.J. Fialho, Worst-case analysis of nonlinear systems, IEEE Trans. Automat. Control 44(6) (1999), pp. 1180-1196.

[4] S. Jayasuriya, On the determination of the worst allowable persistent bounded disturbance for a system with constraints, ASME J. Dyn. Syst. Meas. Control 117 (1995), pp. 126-133.

[5] W.H. Ma, Worst-case evaluation methods for vehicles and vehicles control systems, PhD. Diss., Department of Mechanical Engineering and Applied Mechanics, University of Michigan, Ann Arbor, 1998.

[6] A.Y. Ungoren, Worst-case evaluation methods for rollover prevention system, PhD. Diss., Department of Mechanical Engineering and Applied Mechanics, University of Michigan, Ann Arbor, 2003.

[7] Y. Kou and H. Peng, Development of an integrated chassis control system for worst-case studies, proceedings of AVEC06 8th International Symposium on Advanced Vehicle Control, 2006.

[8] C. Unsal and P. Kachroo, Sliding mode measurement feedback control for antilock braking systems, IEEE Trans. Control Syst. Technol. 7(2) (1999), pp. 271-281.

[9] B. Johansson, Un-tripped SUV rollover detection and prevention, Department of Automatic Control Lund Institute of Technology. ISSN 0280-5316, February 2004.

[10] K. Hayes Suspension control apparatus - US Patent 5924703, May 20, 1997.

[11] A.T. van Zanten, Bosch ESP system:5 years of experience, SAE Conference, SAE Paper No. 2000-01-1633.

[12] C. Buskensa and H. Maurer, SQP-methods for solving optimal control problems with control and state constraints: adjoint variables, sensitivity analysis and real-time control, J. Comput. Appl. Math. 120 (2000), pp. 85-108.

[13] C. Audet and J.E. Dennis Jr., Mesh adaptive direct search algorithm for constrained optimization, SIAM J. Optim. Arch. 17(1) (2006), pp. 188-217.

[14] National Highway Traffic Safety Administration (NHTSA), DOT. Final rule. Federal motor vehicle safety standards electronic stability control systems controls and displays, 2007. 